\title{
La formation en techniques de laboratoire médical au Québec, 1943-1968 : Vingt-cinq ans avant le Cégep
}

\section{Andrée Dufour}

Centre interuniversitaire d'études québécoises, Université Laval

Les progrès marquants de la médecine occidentale au XIX ${ }^{e}$ siècle vont être notamment caractérisés par le développement de nouvelles méthodes de diagnostic des maladies. Parmi ces méthodes, les analyses de laboratoire y tiennent une place encore bien modeste, mais leur l'importance, au Québec notamment, s'accroît considérablement durant le $\mathrm{XX}^{\mathrm{e}}$ siècle. Ces analyses devenant plus complexes et davantage prescrites par le corps médical, il devient même rapidement nécessaire de former un personnel spécialisé, les techniciens de laboratoire.

Ce court texte évoque la formation théorique et pratique de ces nouveaux professionnels de la santé, et ce, de 1943 à 1968. Il se penche sur la nature et la durée des études que requiert la profession, sur le personnel enseignant et sur la composition de la clientèle étudiante. Il fait aussi succinctement état du passage d'une formation donnée à l'université et dans les hôpitaux pendant 25 ans à celle dispensée, depuis la fin des années 1960, dans les collèges d'enseignement professionnel et technique, soit les cégeps.

\section{La nécessité de former un personnel de laboratoire}

Les premières analyses biomédicales, effectuées en milieu hospitalier, pour mieux diagnostiquer et traiter les maladies remontent au XIX siècle au Québec. Un premier petit laboratoire était en effet mis sur pied à l'Hôtel-Dieu de Québec en 1882. Sept autres furent ensuite établis entre 1900 et 1932, à Québec et à Montréal principalement. Les médecins eux-mêmes effectuaient alors les analyses biochimiques les plus simples; les analyses de bactériologie ou d'hématologie, plus complexes, étaient confiées à des laboratoires municipaux.

Cependant le développement des connaissances biomédicales et la demande croissante de soins de santé amènent graduellement les médecins à former 
eux-mêmes un personnel en mesure de les aider dans leurs analyses de laboratoire. Il s'agissait surtout d'infirmières ou parfois même de secrétaires. Cette formation était fort peu organisée et normalisée.

Les années 1940, et surtout 1950, sont toutefois notamment marquées par un rapide élargissement des connaissances biomédicales, par une spécialisation de plus en plus poussée des soins médicaux et par un accroissement spectaculaire de la demande dans le secteur de la santé. Ces transformations poussent le corps médical à se décharger de la responsabilité des analyses aux mains d'un personnel paramédical expressément formé pour œuvrer en laboratoires hospitaliers : les techniciens de laboratoire médical. Il s'agissait d'une nouvelle profession dont la formation, plus courte et moins poussée que la médecine, reposerait toutefois sur une scolarité de niveau postsecondaire. Les femmes y trouveront une possibilité de carrière autre que celles d'infirmière et d'institutrice.

L'École de technologie médicale de l'Université de Montréal se chargea la première de cette formation. Celle-ci fut d'abord donnée en milieu hospitalier, à l'Hôpital Saint-Jean-de-Dieu dirigé, comme maints hôpitaux de l'époque, par une communauté religieuse : les Sœurs de la Providence en l'occurrence. Les exigences d'admission à cette école étaient relativement peu élevées : une $12^{\mathrm{e}}$ année d'études suffisait. Au départ, la scolarité était tout de même longue, d'une durée de quatre ans, car elle comprenait des cours de culture générale : cours de langues, de psychologie, de philosophie, de sociologie, d'économie politique, de christianisme et d'apologétique, lesquels totalisaient $14 \%$ du programme d'études. Les cours de sciences occupaient quelque $30 \%$ de la formation et les stages plus de la moitié du programme. Il reste qu'au terme de leur formation, les candidats et candidates se voyaient octroyer non pas un baccalauréat, mais un diplôme.

En 1953, la formation des techniciens et techniciennes de laboratoire, passe de l'Hôpital Saint-Jean-de-Dieu à l'université, aux mains des médecins plus précisément. Leur formation est réduite à deux années d'études, les matières de culture générale étant pratiquement disparues : elles ne représentent plus qu'un maigre $1 \%$ de la formation. L'apprentissage est devenu nettement plus technique et plus directement relié à l'exercice de la profession.

Sans doute l'Université de Montréal entend-elle imiter la Faculté de médecine de Québec qui, en 1951, a démarré à son École de technologie médicale un programme d'une durée de deux ans dans le but de donner une formation beaucoup plus pratique, spécialisée et technique. La première année du programme d'études est suivie à la Faculté et consiste en une année de formation théorique et de travaux effectués en laboratoire. La seconde année consiste uniquement en des stages accomplis dans les laboratoires des hôpitaux agréés.

\section{À l'Université Laval : deux années d'études très chargées}

La première année de formation donnée à Québec au cours de la décennie 1950 comprend au total plus de 1000 heures de cours et nécessite un nombre important de semaines d'enseignement. Ainsi la session d'automne commence au début du 
mois de septembre et se termine en décembre, quelques jours avant la fête de Noël; quant à la session d'hiver, elle débute immédiatement après la fête de l'Épiphanie et s'étend jusqu'à la mi-juin. Au total, la formation théorique et pratique donnée à l'Université Laval exige 16 semaines de cours à l'automne et 22 ou 23 semaines à l'hiver. C'est largement plus que les 15 semaines de cours par session que comportent bien souvent aujourd'hui les programmes de formation universitaire au Québec.

En seconde année de son programme d'études, la clientèle étudiante effectue des stages en laboratoire d'hôpital durant une année complète, effectués le plus souvent en bactériologie, en chimie clinique, en hématologie, en histopathologie et en sérologie.

Au total, la charge d'études que comportent les cours théoriques, les travaux pratiques et les stages hospitaliers est considérable. De fait, le transfert du programme de technologie médicale de l'hôpital à l'université et le passage de quatre ans à deux ans de formation n'ont pas signifié la diminution du nombre d'heures d'études requises. Au contraire, elles ont augmenté de $30 \%$. C'est énorme en comparaison de certains programmes universitaires, tels que les programmes de diététique et de lettres, fréquentés par les jeunes Québécoises. Les étudiantes en technologie médicale — et aussi les étudiants même s'ils sont beaucoup moins nombreux — doivent fournir durant leurs deux années d'études, un nombre d'heures de cours et de stages équivalent à celui que les étudiantes inscrites au programme de l'Institut de diététique fournissent, elles, en quatre ans. Et ce, sans pouvoir bénéficier du statut plus élevé dont jouit la diététique.

\section{Contenu de la formation donnée à l'université}

Transférée ou directement établie à l'université sous le contrôle des médecins, la formation des futurs techniciens et techniciennes donnée durant la première année d'études est très variée en plus d'être très dense. Ainsi, à l'Université Laval, selon les Annuaires de l'École de technologie médicale publiés par l'Extension de l'enseignement de cette université, elle comprend 310 heures de cours théoriques et 695 heures de cours pratiques. La culture générale, qui faisait partie du programme lorsque celui-ci était donné à Montréal en milieu hospitalier, a disparu. Mais les cours théoriques abordent de nombreuses autres matières : l'anatomie, la physiologie, la biochimie, la biochimie médicale, la chimie, la chimie clinique, la cytopathologie, le dessin, l'électrocardiographie, l'éthique professionnelle et morale, l'hématologie, l'histopathologie, les mathématiques, le métabolisme de base, la microbiologie et la sérologie, la physique et les statistiques. Les cours d'anatomie et de physiologie, de biochimie, de chimie, de chimie clinique, d'éthique professionnelle et morale, de microbiologie et de sérologie occupent la majorité des heures de cours théoriques. Les cours théoriques, qui touchent de nombreuses matières en un nombre d'heures somme toute limité, ne permettent pas la transmission d'un savoir théorique spécialisé. 


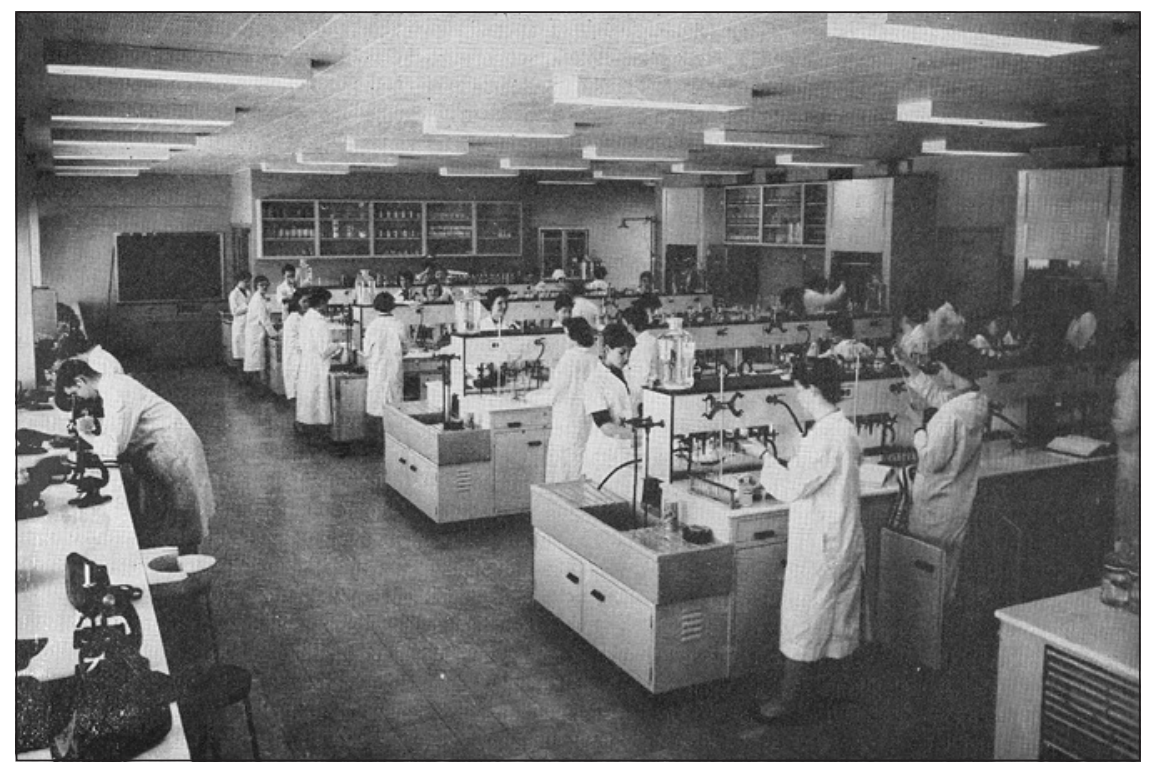

Figure 1. Groupe d'étudiantes et d'étudiants au laboratoire. Université Laval. Extension de l'enseignement. Ecole de Technologie Médicale. Annuaire 1967-1968.

Les 695 heures de travaux pratiques effectués en laboratoire concernent les différentes matières théoriques, à l'exception des mathématiques, de la physique, des statistiques et de l'éthique professionnelle et morale. Le dessin n'est enseigné que sous la forme d'exercices pratiques.

\section{Le corps enseignant}

À Montréal, lorsque le programme de technologie médicale était donné à l'Hôpital Saint-Jean-de-Dieu, les Sœurs de la Providence se chargeaient assurément d'une bonne partie de la formation générale, des cours de christianisme et d'apologétique notamment. Les cours de psychologie, de philosophie, de sociologie et d'économie politique étaient probablement donnés par les religieuses ou par des spécialistes de ces disciplines.

Au début des années 1960, les données disponibles montrent que huit ans après le transfert de l'école à l'Université de Montréal, les femmes enseignantes, des techniciennes médicales en majorité, sont largement minoritaires. Qui plus est, elles occupent surtout des fonctions d'auxiliaires d'enseignement, chargées de travaux pratiques, des démonstrations en laboratoire. À l'Université Laval, les annuaires de l'École de technologie médicale publiés chaque année par le service de l' "Extension de l'enseignement ", font état du corps professoral. Celui de 1967-1968, peu modifié depuis la fondation de l'école, permet de constater que 16 professeurs, tous masculins et presque tous médecins, assurent la formation théorique donnée durant la première année du programme. Des diplômées en technologie médicale d'expérience, des 
femmes en majorité, font office de " démonstrateurs » et assurent l'encadrement des travaux pratiques en laboratoire. Lors des stages dans les hôpitaux, ce sont également des techniciens ou des techniciennes qui assurent la formation des stagiaires, sous la supervision, éloignée, des médecins responsables des différents laboratoires.

\section{La clientèle étudiante}

Lorsque la technologie médicale était dispensée par les Sœurs de la Providence à l'Université de Montréal, les jeunes hommes représentaient une importante partie des inscriptions à ce programme d'études. Ainsi, entre 1951 et 1953, soit peu avant le passage du programme à l'université, les inscriptions masculines s'élevaient jusqu'à $50 \%$ du total des inscriptions.

En 1955, la présence masculine aux cours est réduite à un maigre $1 \%$. Le programme d'études a donc connu une féminisation extrêmement rapide, quelque peu difficile à expliquer. Aussi les historiennes Nadia Fahmy-Eid et Aline Charles se demandent-elles si la " dévalorisation du programme de technologie médicale, qui passe d'un baccalauréat de quatre ans à un diplôme de deux ans " a pu constituer " une explication suffisante "? Le phénomène est d'autant plus étonnant que la formation théorique est donnée dorénavant par des hommes laïques. Et il durera : jusqu'au transfert du programme d'études dans les cégeps en 1971, les filles seront en nette majorité, les garçons ne composant que tout au plus $8 \%$ de la clientèle. Il y a aussi le fait que la courte durée du programme de technologie médicale, désormais parrainé par l'université, attire particulièrement les filles dont bon nombre-nous évoquons ici les années 1950 et le tout début des années 1960 — songent peut-être au mariage et ne veulent pas dès lors consacrer un trop grand nombre d'années à l'apprentissage d'une profession qu'elles pourraient rapidement quitter avec l'arrivée des enfants. Pour cette raison, les garçons sont recherchés. Certains hôpitaux n’hésitent pas à leur offrir des conditions salariales intéressantes et des postes de responsabilité pour les retenir après leur stage dans les différents laboratoires de l'institution ou pour les faire venir d'autres hôpitaux.

\section{Réussite et diplomation}

Le programme universitaire de technologie médicale d'une durée de deux ans seulement attire bon nombre de candidatures alors que les places sont limitées en raison notamment des locaux et du matériel que nécessitent les travaux en laboratoire et de la capacité des hôpitaux à accueillir des stagiaires. Les autorités responsables du programme imposent donc un examen d'entrée qui réduit très largement la cohorte étudiante. Ainsi le pourcentage de réussite du programme d'études est-il élevé (Tableau 1).

Le taux de diplomation des étudiants et étudiantes est élevé et contribue sans doute à l'augmentation de l'effectif de la profession. Selon l'étude de R. Béland et T. Boudreau, La prévision de la main-d'euvre dans le secteur hospitalier réalisée en 1970 dans le cadre de la commission Castonguay-Nepveu, le Québec comptait 378 technologistes médicaux en 1956. Dix ans plus tard, leur nombre avait quadruplé : 1583 
technologistes médicaux œuvraient en laboratoire dans les différents hôpitaux du Québec. De fait il s'agit d'une progression importante qui s'inscrivait dans l'intervention accrue de l'État en matière de santé et qui dépassait même celle des infirmières dont le nombre était passé durant les mêmes années de 5116 à 14868 .

Tableau 1

Taux de diplomation des étudiants-tes en technologie médicale à l'Université de Montréal, 1957-1964

\begin{tabular}{|c|c|c|c|}
\hline $\begin{array}{c}\text { Année de } \\
\text { diplomation }\end{array}$ & $\begin{array}{c}\text { Nombre } \\
\text { d'inscriptions }\end{array}$ & $\begin{array}{c}\text { Nombre } \\
\text { de diplômé-es }\end{array}$ & $\begin{array}{c}\text { Pourcentage } \\
\text { de réussite }\end{array}$ \\
\hline 1957 & 43 & 31 & $72 \%$ \\
\hline 1958 & 35 & 33 & $95 \%$ \\
\hline 1959 & 39 & 31 & $79 \%$ \\
\hline 1960 & 47 & 36 & $76 \%$ \\
\hline 1961 & n.d. & 41 & $100 \%$ \\
\hline 1962 & 55 & 55 & $83 \%$ \\
\hline 1963 & 65 & 54 & $90 \%$ \\
\hline 1964 & 68 & 61 & d. \\
\hline
\end{tabular}

Source : Statistiques du registraire de l'Université de Montréal (Fahmy-Eid et Charles, 1988 : 21)

\section{La fin d'une formation universitaire}

Axée sur l'acquisition d'un savoir pratique, la technologie médicale participe au processus du diagnostic-traitement. Toutefois la formation acquise à l'université, à l'École de technologie médicale, définie par le corps médical, ne fait que peu de place à un savoir théorique spécialisé. Aussi, comme l'estiment Nadia Fahmy-Eid et Lucie Piché, les technologistes médicales n’obtiennent-elles qu'un statut professionnel secondaire par rapport à la médecine. ${ }^{2}$

À la suite de la commission Parent et de la création des cégeps, la formation universitaire des technologistes médicaux cède la place à un programme de niveau collégial de trois ans au début des années 1970. Le volet théorique de la formation qui comprend, comme chez les Sœurs de la Providence, des cours de culture générale, de sciences et de travaux pratiques, est désormais donné dans ces institutions d'enseignement alors que les stages continuent toutefois d'être effectués en milieu hospitalier dans la plupart des cas, même si des stagiaires peuvent être acceptés dans un institut de recherche notamment. 


\section{Notes}

1 Nadia Fahmy-Eid et Aline Charles, « Savoir contrôlé ou pouvoir confisqué? La formation des filles en technologie médicale, réhabilitation et diététique à l'Université de Montréal (1940-1970) ", Recherches féministes 1, 1 (1988) : 19.

2 Nadia Fahmy-Eid et Lucie Piché, "Le savoir négocié. Les stratégies des associations de technologie médicale, de physiothérapie et de diététique pour l'accès à une meilleure formation professionnelle (1930-1970) "Revue d'histoire de l'Amérique française 43, 4 (1990) : 509-534; Nadia Fahmy-Eid et Lucie Piché, "À la recherche d'un statut professionnel dans le champ paramédical. Le cas de la diététique, de la physiothérapie et de la technologie médicale (1940-1973) ", Revue d'histoire de l'Amérique française 45, 3 (1992) : 375-401. 